\title{
ESTRATÉGIAS DE PRÉ-CONCENTRAÇÃO EM ELETROFORESE CAPILAR (CE). PARTE 1. MANIPULAÇÃO DA VELOCIDADE ELETROFORÉTICA DO ANALITO
}

\author{
Maria de Lourdes Leite de Moraes* \\ Departamento de Ciências Exatas e da Terra, Universidade Federal de São Paulo, Rua Artur Riedel, 275, 09972-270 Diadema - \\ SP, Brasil
}

\section{Solange Leite de Moraes}

Departamento de Física e Química, Faculdade de Ciências Farmacêuticas de Ribeirão Preto, Universidade de São Paulo, Av. do Café, s/n, 14040-903 Ribeirão Preto - SP, Brasil

\section{Elisabete Alves Pereira}

Universidade Federal de São Carlos, Campus Sorocaba, CP 3031, 18043-970 Sorocaba - SP, Brasil

Marina Franco Maggi Tavares

Departamento de Química Fundamental, Instituto de Química, Universidade de São Paulo, Av. Lineu Prestes, 748, 05508-900 São Paulo - SP, Brasil

Recebido em 6/4/08; aceito em 23/10/08; publicado na web em 2/4/09

\begin{abstract}
PRECONCENTRATION STRATEGIES IN CAPILLARY ELECTROPHORESIS (CE). PART 1. MANIPULATION OF THE ANALYTE ELECTROPHORETIC VELOCITY. Capillary electrophoresis has become a well-established and routine-based separation technique. It is based on the differences between charged analyte mobility in aqueous or organic electrolytes. Its major limitation is the sensitivity due to small sample injection volumes and the narrow diameter of the capillaries, especially when UV detection is used. There are a number of ways to increase the concentration sensitivity. This report shows some on-line preconcentration strategies to perform it in free solution capillary electrophoresis that are based on manipulation of the analyte electrophoretic velocity during the sample introduction (stacking, field amplification and transient isotachophoresis).
\end{abstract}

Keywords: capillary electrophoresis; preconcentration strategies; stacking.

\section{INTRODUÇÃO}

A eletroforese capilar (CE, capillary electrophoresis) é uma técnica extremamente útil para separar compostos iônicos ou ionizáveis devido a sua alta eficiência de separação. ${ }^{1-5} \mathrm{O}$ uso de capilares de sílica fundida com diâmetro interno da ordem de micrometros contribuiu com inúmeras vantagens à técnica, mas também foi responsável pela sua maior limitação: a baixa sensibilidade de detecção, particularmente para compostos que absorvem no ultravioleta (UV, ultra-violet) ${ }^{4,5}$ Isto ocorre devido ao reduzido caminho óptico definido pelo diâmetro do capilar (entre 50 e $75 \mu \mathrm{m}$ ), associado ao pequeno volume de amostra injetado (da ordem de nanolitros). ${ }^{1}$

Nos últimos anos, o desenvolvimento de estratégias on-line para pré-concentrar e/ou aumentar a quantidade de amostra que pode ser inserida na coluna, sem afetar a eficiência da separação, tem recebido cada vez mais atenção por parte dos pesquisadores. ${ }^{6-28} \mathrm{Em}$ eletroforese capilar em solução livre (FSCE, Free solution capillary electrophoresis), estas estratégias de pré-concentração são baseadas nos fenômenos físico-químicos usados para pré-concentrar os analitos e envolvem a manipulação da composição da amostra (através da manipulação da velocidade eletroforética do analito) e do tampão ou eletrólito de corrida (BGE, background electrolyte), juntamente com procedimentos de injeção simples, sem necessidade de alterar a instrumentação presente..$^{15}$ Estas estratégias são conhecidas como sample stacking ou stacking e podem proporcionar de 10 a mais de 1000 vezes de melhora na resposta do detector. ${ }^{6-15}$ As estratégias de stacking incluem os modos: stacking mediado por força iônica, ${ }^{15,20-}$ 22 stacking em campo amplificado, ${ }^{10,11,23-25}$ stacking em injeção de

*e-mail: mllmoraes@unifesp.br grande volume, ${ }^{11,26,27}$ stacking mediado por $\mathrm{pH}^{9,16,28-32} \mathrm{e}$ isotacoforese transiente. ${ }^{17-19,33-36}$

Neste trabalho são apresentados e discutidos os conceitos e modos de se realizar as estratégias de pré-concentração on-line que envolvem a manipulação da velocidade eletroforética do analito.

\section{Stacking mediado por força iônica}

A estratégia mais simples para pré-concentrar amostras é o stacking mediado por força iônica, que foi apresentado pela primeira vez, em 1979, por Mikkers e colaboradores. ${ }^{20}$ Nesta estratégia, um grande volume de amostra, preparada em solvente com condutividade elétrica menor que a do eletrólito de corrida, é introduzido hidrostaticamente no capilar (Figura 1A). Após a aplicação da tensão, esta região de baixa condutividade experimentará um campo elétrico $(E)$ maior que a região do eletrólito de corrida, pois a resistividade na banda da amostra é maior que a resistividade no restante da coluna, gerando um alto campo elétrico. Os íons presentes na amostra movem-se mais rapidamente na região de baixa condutividade que na região de alta condutividade. Quando os íons alcançam a interface entre a banda da amostra (alto campo elétrico local) e a banda do eletrólito de corrida (baixo campo elétrico local), são desacelerados, causando uma contração da banda da amostra (Figura 1B). Ocorre, então, uma redução do comprimento da banda da amostra, provendo uma região com concentração maior que a original. O fluxo eletrosmótico (eof, electroosmotic flow) neste ponto é considerado zero. ${ }^{15} \mathrm{O}$ campo elétrico torna-se então homogêneo e a amostra é separada segundo os princípios convencionais da eletroforese capilar (Figura 1C). ${ }^{11}$ $\mathrm{O}$ mecanismo de stacking ocorre tanto para as espécies carregadas positivamente quanto para as espécies carregadas negativamente. 
As espécies positivas concentram-se na frente da banda da amostra, enquanto as espécies negativas se concentram na parte de trás.

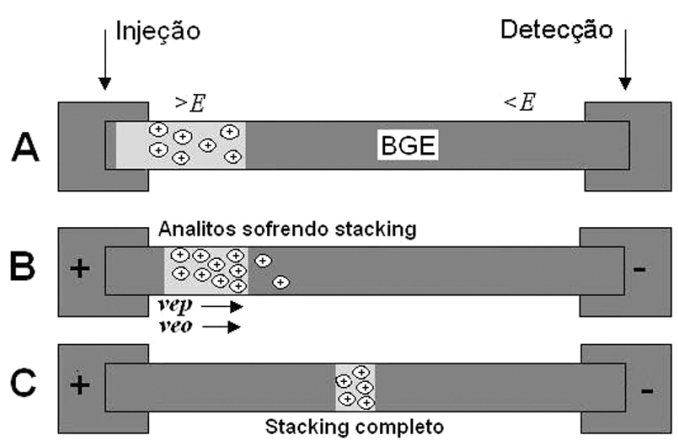

Figura 1. Esquema representativo do stacking mediado por força iônica. $E=$ campo elétrico, $v_{e p}=$ velocidade eletroforética, $v_{e o}=$ velocidade eletroosmótica, $B G E=$ eletrólito de corrida

Burgi e Chien ${ }^{21}$ descreveram um modelo para otimizar o stacking e discutiram as limitações da eficiência desta estratégia. Teoricamente, a extensão com a qual o stacking ocorre é proporcional à resistividade (ou ao inverso da condutividade) entre o eletrólito na região da amostra e o eletrólito no restante da coluna. Estas diferenças entre as condutividades na banda da amostra e no eletrólito podem ser expressas quantitativamente como: $:^{(11,21)}$

$\frac{E_{1}}{E_{2}}=\frac{\rho_{1}}{\rho_{2}}=\frac{C_{2}}{C_{1}}=\gamma$

onde $C_{1}$ e $C_{2}$ são as concentrações do eletrólito (ou tampão) dentro da banda de amostra e no restante da coluna, respectivamente; $E_{1}$ e $E_{2}$, o campo elétrico nestas regiões; $\rho_{1 \mathrm{e}} \rho_{2}$, as resistividades respectivas e $\gamma$ o fator de enriquecimento de campo.

Em geral, as resistividades são inversamente proporcionais à concentração do eletrólito (para tampões com a mesma composição). No caso de amostras preparadas em água ou em tampão com concentração muito diluída, os efeitos de impurezas e dos íons da amostra sobre a resistividade total devem ser considerados. Para amostras preparadas em água, o fator de enriquecimento de campo pode ser da ordem de centenas de vezes. ${ }^{21}$

Normalmente, em CE a amostra é preparada no próprio tampão de corrida e, neste caso, não há diferença de condutividade entre as duas bandas (da amostra e do tampão). Para que o stacking seja obtido, a banda de amostra deve necessariamente ter uma condutividade menor que o tampão de corrida. Sendo assim, a amostra deve ser preparada ou num eletrólito que seja 10 vezes menos concentrado que o eletrólito de corrida ${ }^{21}$ ou em água; ou pode-se ainda adicionar um solvente orgânico na amostra como, por exemplo, acetonitrila. ${ }^{11,22}$

Uma vez que o fluxo de íons que passa através do capilar deve ser constante dentro de cada banda, a concentração no estado de equilíbrio dos solutos $\left[\mathrm{S}_{1}\right]$ e $\left[\mathrm{S}_{2}\right.$ ] é inversamente proporcional à força do campo elétrico e, por essa razão, sua velocidade eletroforética (vep) é:

$\left[\mathrm{S}_{1}\right] \mathrm{V}_{\mathrm{ep} 1}=\left[\mathrm{S}_{2}\right] \mathrm{V}_{\mathrm{ep} 2}$

onde $\left[\mathrm{S}_{1}\right]$ e $\left[\mathrm{S}_{2}\right]$ são as concentrações do soluto dentro da banda da amostra e no restante da coluna, respectivamente, e $\mathrm{v}_{\mathrm{ep} 1} \mathrm{e}_{\mathrm{ep} 2}$ são as velocidades eletroforéticas nestas regiões.

Como a velocidade eletroforética dos solutos é proporcional ao campo elétrico, a concentração do analito é dada por:
$\frac{\left[S_{2}\right]}{\left[S_{1}\right]}=\frac{C_{2}}{C_{1}}=\gamma$

A Equação 3 mostra que a concentração dos íons da amostra (que migram dentro da região do eletrólito de corrida) aumenta pelo fator de enriquecimento de campo. Devido à conservação da massa, o comprimento da banda de amostra na região do eletrólito deve diminuir (comprimir) pelo mesmo fator. Negligenciando os efeitos de difusão, o comprimento da banda de amostra após o stacking seria então dado por:

$X_{s}=\frac{X_{i}}{\gamma}$

onde $X_{\mathrm{s}}$ é o comprimento da banda da amostra na região do eletrólito após o stacking e $X_{\mathrm{i}}$ o comprimento inicial da banda de amostra injetada.

Contudo, se ocorrerem grandes diferenças de concentração entre as duas bandas (por exemplo, preparar a amostra em água ou em um eletrólito de concentração muito baixa e utilizar um eletrólito de corrida com uma alta concentração) pode ocorrer alargamento das bandas pré-concentradas devido ao fluxo laminar. ${ }^{21}$ Porém, esta perturbação sobre o stacking é muito pequena devido à alta velocidade dos íons da amostra na banda de injeção.

\section{Stacking em campo amplificado}

A pré-concentração em campo amplificado é similar ao stacking mediado por força iônica. ${ }^{4,6}$ Nesta estratégia, a amostra é preparada num solvente de baixa condutividade (ou água) e é injetada eletrocineticamente, aplicando-se tensão positiva ou negativa, dependendo da carga do analito. Antes da introdução da amostra, uma banda de água é introduzida hidrodinamicamente no capilar para garantir as condições de stacking em campo elétrico elevado ${ }^{7}$ (Figura 2A). Quando a tensão é aplicada, forma-se uma região de alto campo elétrico na banda de água, que é maior que no restante da solução. Os íons migram através da banda da amostra e são acelerados na região da banda de água. Quando estes íons ultrapassam o limite entre a banda de água e o elétrolito de corrida, encontram um campo elétrico menor e são desacelerados, concentrando-se em uma banda fina no limite entre estas duas regiões (Figura 2B). ${ }^{11,23}$ Assim que os campos elétricos são igualados, os analitos são separados normalmente (Figura 2C). A injeção desta pequena banda de água garante um elevado campo elétrico local, ou seja, no ponto de injeção. ${ }^{24}$

Uma vantagem desta estratégia é a possibilidade de injeção seletiva, ${ }^{12}$ otimizando o $\mathrm{pH}$ da amostra. É possível melhorar a seletividade e a velocidade de separação pela introdução de espécies exclusivamente catiônicas ou aniônicas. A inclinação dos picos esperada para injeção eletrocinética é reduzida (porém não eliminada) e podem ser empregadas tensões maiores na injeção. A injeção eletrocinética apresentou um ganho de 200 vezes comparada à injeção hidrodinâmica, neste tipo de estratégia. ${ }^{25}$

O mecanismo de compressão do pico é similar ao stacking mediado por força iônica. A banda de água diferencia a forma da compressão do pico em relação ao stacking convencional. O comprimento da banda de amostra injetada (Xi) pode ser estimado por: ${ }^{11}$

$X i=\left(\frac{\mu_{e o}}{r}+\mu e p\right) E t$

onde $r$ é razão das resistividades do eletrólito de corrida e a solução de amostra injetada, E é o campo elétrico, $\mu_{e o}$ é a mobilidade ele- 
troosmótica, $\mu_{e p}$ é a mobilidade eletroforética, $t$ é o tempo de injeção da amostra.

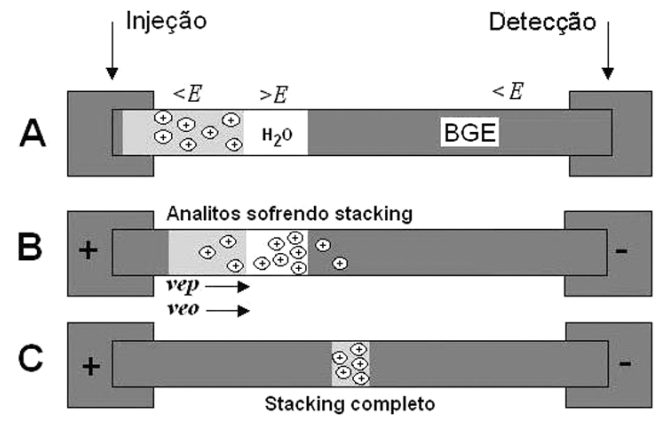

Figura 2. Esquema representativo do stacking em campo amplificado. Legenda igual à da Figura 1

\section{Stacking em injeção de grande volume}

Quando se trabalha com amostras muito diluídas, algumas vezes é necessário injetar um volume maior de amostra (aproximadamente 2/3 do capilar) para garantir a sensibilidade de detecção no UV. Neste caso, o campo elétrico através do capilar muda, mas o campo elétrico total permanece constante. ${ }^{11}$ Como a força do campo elétrico está concentrada praticamente na banda da amostra, o campo elétrico no eletrólito de corrida é praticamente zero. Assim, quando os solutos alcançam o eletrólito de corrida, sua velocidade chega quase a zero e eles são conduzidos pelo fluxo eletrosmótico, o qual é dominado pela velocidade eletrosmótica da banda de amostra. Como conseqüência, os solutos não são separados. A melhor separação, neste caso, é obtida quando o comprimento da banda de amostra é menor que $1 \%$ do comprimento total do capilar. ${ }^{11,26}$

Quando são introduzidos grandes volumes de amostra também ocorrem diferenças significativas na condutividade entre a banda da amostra e o elétrolito de corrida, o que gera altas temperaturas na banda da amostra. ${ }^{27}$ Altas temperaturas podem causar decomposição térmica de alguns solutos e até mesmo efervescência da amostra. ${ }^{23}$ Assim, quanto maior o volume injetado, maior é o alargamento das bandas de acordo com a equação:

$V_{\text {inj }}=Q V_{\text {coluna }} / N^{1 / 2}$

onde $V_{i n j}$ é o volume de injeção, $V_{\text {coluna }}$ é o volume da coluna, $Q$ é a fração de alargamento de pico permitida e $N$ o número de pratos teóricos para a separação.

Para resolver os problemas de decomposição térmica, Chien e Burgi $^{8}$ desenvolveram uma estratégia de stacking para injeções de grandes volumes. Este tipo de stacking é demonstrado para solutos catiônicos. Todo o capilar ou uma grande parte dele é preenchido com a amostra que é introduzida por pressão (Figura 3A). O fluxo eletrosmótico é invertido adicionando-se um aditivo ao tampão de corrida como, por exemplo, brometo de cetiltrimetilamônio (CTAB, cetyl trimethylammonium bromide) ${ }^{1,11}$ Quando a tensão positiva é aplicada no pólo de injeção, os íons positivos da amostra migram (com velocidade eletroforética) para o detector, enquanto o tampão entra no capilar pelo lado oposto (lado do detector). Porém, como o fluxo eletrosmótico é sempre de magnitude maior que o fluxo eletroforético, os íons são empurrados de volta ao ponto de injeção, sendo concentrados numa banda fina na fronteira entre a banda da amostra e o eletrólito de corrida (Figura 3B). Isto pode ser acompanhado pela leitura da corrente elétrica. Quando o valor da corrente atinge 95-99\% do valor inicial da corrente do eletrólito de corrida (medida anterior- mente), a polaridade é invertida no pólo de injeção e o tampão passa a entrar no capilar pelo lado da injeção. Os analitos pré-concentrados são então separados normalmente ${ }^{11}$ (Figura 3C).

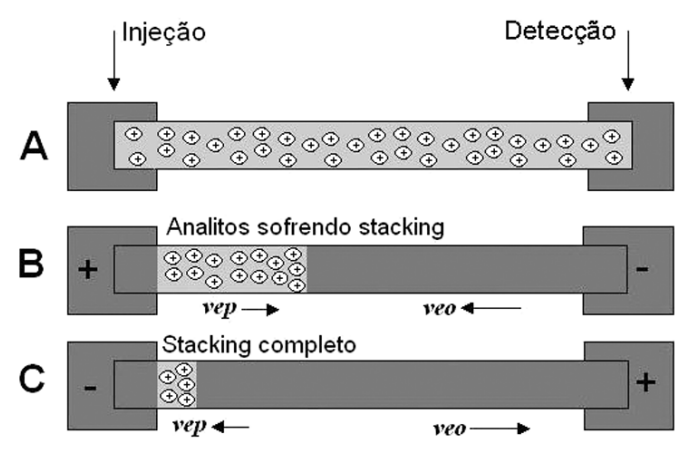

Figura 3. Esquema representativo do stacking por injeção em grande volume. Legenda igual à da Figura 1

\section{Stacking mediado por $\mathrm{pH}$}

O stacking mediado por $\mathrm{pH}$ tem sido utilizado para separar amostras com alta força iônica ou quando se deseja separar compostos anfóteros. ${ }^{11,28-32} \mathrm{O}$ stacking ácido ${ }^{28}$ é usado para analitos catiônicos e o stacking básico ${ }^{29}$ para analitos aniônicos. $\mathrm{Na}$ análise de compostos aniônicos, uma banda diluída de amostra é injetada no capilar usando o modo de injeção eletrocinético ou hidrodinâmico, formando uma região de baixo pH. Imediatamente após a injeção da amostra, uma banda de um composto com $\mathrm{pH}$ básico (por exemplo, hidróxido de sódio ou amônio) é injetada eletrocineticamente no capilar formando uma região de alto $\mathrm{pH}$ (Figura 4A). A base titula o eletrólito de corrida (BGE) resultando na formação de uma banda de alto campo elétrico (baixa condutividade). Isto faz com que o analito (na forma aniônica) pré-concentre na interface entre a banda de baixa condutividade e o BGE, resultando no stacking da amostra dentro de uma banda fina $^{30}$ (Figura 4B). Terminada a injeção do hidróxido o stacking está completo e a separação se inicia. A banda de baixa condutividade se dissipa e o BGE no capilar se torna novamente homogêneo ${ }^{28,29}$ (Figura 4C).

Deve haver uma relação ótima entre a quantidade da amostra injetada e a quantidade de base introduzida no capilar, sendo que o tempo de injeção ótimo da base é função do comprimento do capilar, da força iônica da amostra e do eletrólito, e da mobilidade eletroforética do analito. ${ }^{31}$ Portanto, o stacking é função do tempo de injeção da base. A vantagem desta estratégia é que um grande volume de amostra altamente iônica pode ser injetada diretamente sem nenhum tratamento prévio. ${ }^{31,32}$

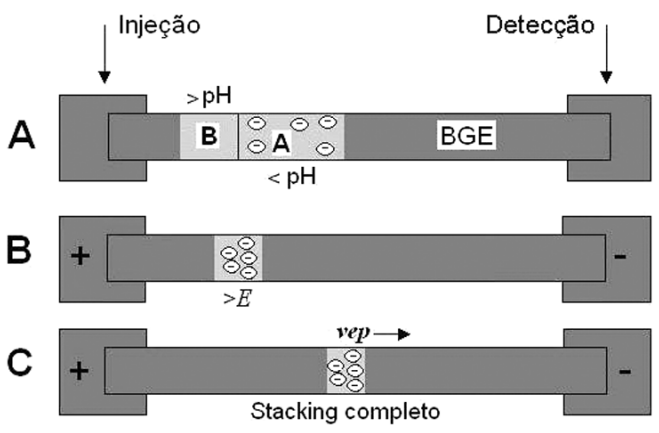

Figura 4. Esquema representativo do stacking medidado por $\mathrm{pH}$. Legenda igual à da Figura 1. B = base, $A=$ Amostra 


\section{Isotacoforese transiente}

A isotacoforese transiente (tITP, transient isotachophoresis) é um método de pré-concentração que combina a isotacoforese (ITP, isotachophoresis) e a eletroforese capilar de zona (CZE, capillary zone electrophoresis) em um único capilar. ${ }^{33} \mathrm{~A} \mathrm{ITP-CZE} \mathrm{(ou} \mathrm{tITP)} \mathrm{foi}$ reportada pela primeira vez por Verheggen e colaboradores,${ }^{34}$ sendo uma estratégia relativamente simples, de fácil automação e aplicável a todos os equipamentos comerciais.

Em ITP, os analitos são separados em bandas sucessivas adjacentes de acordo com a diminuição da sua mobilidade eletroforética. A amostra é introduzida entre um eletrólito chamado líder (LE), que tem mobilidade maior que os analitos da amostra, e um eletrólito chamado terminador (TE), o qual tem a mobilidade menor que os analitos da amostra. ${ }^{31,35}$ Quando a tensão é aplicada, a concentração de cada banda separada é automaticamente ajustada àquela determinada pela concentração do íon nas vizinhanças da banda, que é dada por:

$$
C_{A}=\frac{C_{L} \mu_{A}\left(\mu_{L}+\mu_{Q}\right)}{\mu_{L}\left(\mu_{A}+\mu_{Q}\right)}
$$

onde $\mu_{L}>\mu_{A}$ e $C_{A}, C_{L}$ são as concentrações do analito A na banda adjacente ao eletrolito lider, $\mathrm{L} ; \mu_{A}, \mu_{L}$ e $\mu_{Q}$ são as mobilidades eletroforéticas de A, L e do contra íon Q.

Um dos modos de se realizar a tITP é estabelecer as condições de ITP primeiramente injetando um eletrólito líder (antes da injeção da amostra). O eletrólito terminador é injetado depois da banda de amostra (Figura 5A). Quando a tensão é aplicada, a ITP se inicia e as concentrações de eletrólito líder e terminador vão se adequando a fim de proporcionar uma focalização das bandas. Os analitos, neste caso, são concentrados pelos princípios da $\operatorname{ITP}^{31}$ (Figura 5 B). No caso da tITP, após a etapa de focalização das bandas, o eletrólito terminador é trocado pelo eletrólito de corrida e os analitos são separados por CZE convencional (Figura 5C). Os componentes são então préconcentrados durante a ITP e analisados durante a CZE.

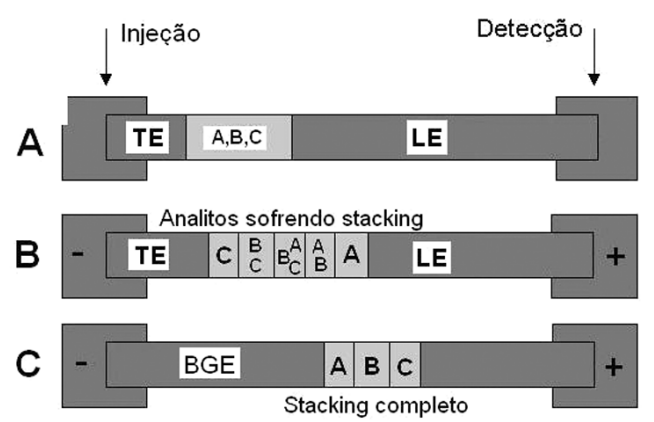

Figura 5. Representação esquemática da isotacoforese capilar. A, B e $C=$ analitos representativos da amostra, $L E=$ eletrólito Líder e TE = eletrólito terminador

A combinação destes dois modos de eletroforese pode ser realizada no mesmo capilar ou em capilares acoplados, sendo possível injetar grandes volumes de material diluído. Os capilares acoplados requerem uma instrumentação mais complexa, não sendo utilizados nos sistemas de eletroforese comerciais. Neste caso, a amostra é concentrada no primeiro capilar por isotacoforese e depois injetada no segundo capilar para ser separada por CZE, utilizando um eletrólito convencional de CE. O uso de um único capilar é mais interessante, pois a tITP pode ser realizada em instrumentos convencionais de eletroforese, eliminando os problemas de acoplamento de capilares.
Nesta estratégia, a detectabilidade pode ser superior a $10^{3}$ vezes e o limite de detecção da ordem de $10^{-9} \mathrm{~mol} / \mathrm{L} .^{36}$

Um parâmetro importante com respeito ao tempo de focalização isotacoforética é a quantidade de carga elétrica $\left(Q_{s}\right)$ que passa através da coluna. O tempo de focalização em ITP de dois componentes A e B pode ser relacionado diretamente a $Q s$, dependendo da quantidade de íons introduzidos $(n)$ e das suas mobilidades $(\mu){ }^{36}$

$Q_{s}=\frac{F n a \mu a(1-\mu R / \mu a)+n b \mu b(1-\mu R / \mu b)}{\mu a-\mu b}$

onde $\mu_{a}$ e $\mu_{b}$ representam as mobilidades absolutas ou iônicas dos componentes $A$ e $B, n_{a}$ e $n_{b}$ é a quantidade de íons de cada componente A e B, $F$ é a constante de Faraday e $\mu_{R}$ é a mobilidade absoluta do contra-íon, R.

A carga que passa pela coluna $(Q 1)$, antes da banda da amostra alcançar o detector, é obtida pela expressão:

$Q 1=F n 1(1-\mu R / \mu 1)$

onde $n 1$ é a quantidade de íons do eletrólito líder no capilar.

Existem diferentes maneiras de se estabelecer um sistema com eletrólitos descontínuos para que ocorra a concentração isotacoforética no início da separação. ${ }^{9}$ Uma delas é preencher o capilar com o eletrólito terminador e, antes da injeção da amostra, injetar uma banda de um eletrólito que tenha alta mobilidade (eletrólito líder) (Figura 6A). A concentração da amostra é então adaptada à concentração do eletrólito líder. Como a banda do eletrólito líder (LE) é injetada após uma banda de baixa mobilidade (TE), o LE migra eletroforeticamente na banda do TE e se move para longe dos íons da amostra. Desta forma, os analitos são separados por CZE no eletrólito terminador. ${ }^{36} \mathrm{O}$ arranjo inverso é obtido quando o capilar é preenchido com o líder e a banda do terminador é injetada a seguir, o qual levaria também a uma pré-concentração dos analitos (representação não mostrada).

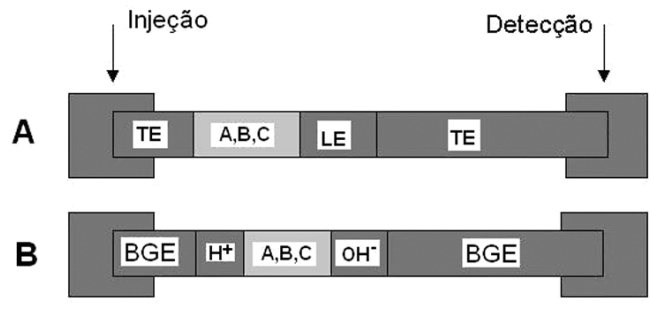

Figura 6. Representação esquemática da isotacoforese transiente: (A) sistema de pré-concentração utilizando dois tampões diferentes, (B) sistema de pré-concentração utilizando um único eletrólito de corrida ou tampão. Representação esquemática da isotacoforese capilar. $A, B$ e $C=$ analitos representativos da amostra, $L E=$ eletrólito Líder e $T E=$ eletrólito terminador. $B G E=$ eletrólito de corrida

Um outro tipo de sistema tampão descontínuo é usado quando uma separação requer um $\mathrm{pH}$ extremo. Neste caso, a amostra é injetada entre uma banda de $\mathrm{OH}^{-}$e $\mathrm{H}^{+}$(Figura 6B). ${ }^{9,36}$ Quando a tensão é aplicada, as bandas de ácido e base migram uma contra a outra, formando uma região de baixa condutividade no meio da banda de amostra, ou seja, o campo elétrico na banda da amostra torna-se maior que no restante da coluna devido à maior resistência. Este campo elétrico descontínuo proporciona uma redução na velocidade dos íons da amostra quando estes cruzam o limite estacionário entre a amostra e o BGE, levando à pré- 
Tabela 1. Comentários gerais para algumas estratégias de aumento de sensibilidade baseadas na manipulação da velocidade eletroforética do analito

\begin{tabular}{ll}
\hline Estratégia & $\begin{array}{l}\text { Aumento em } \\
\text { sensibilidade }\end{array} \quad$ Vantagens
\end{tabular} Desvantagens $\quad$ Aplicações e estudos

Stacking mediado por força iônica

Simples. Pode ser aplicada a cátions e ânions simultaneamente.

Depende da força iônica da

Simples. Possibilidade de

Stacking em campo amplificado

$100-1000$ injeção seletiva.

Requer um maior cuidado.

Stacking em grande volume

$100-1000$ Utilizada para amostras muito diluídas

Simples. Separação de compostos anfóteros e amostras com alta força iônica.

Podem-se injetar grandes volumes de amostra.

Um pouco mais trabalhosa. Fácil automação Aplicável a todos os equipamentos comerciais. amostra em relação ao eletrólito de separação

Não pode ser aplicada a cátions e ânions simultaneamente

Análise de drogas anti-inflamatórias não esteroidais ${ }^{26,37}$ e análise de ácidos fenólicos. ${ }^{38}$

Estudos sobre a utilização de acetonitrila na amostra. ${ }^{10}$ Análises de drogas $^{39,40}$ e compostos básicos. ${ }^{41}$

Podem ocorrer perdas de amostra na etapa de reverter a polaridade. Quanto maior o volume injetado maior o alargamento da banda.

Antibióticos em amostras de água, ${ }^{43}$ drogas catiônicas em amostras de urina, ${ }^{15,42}$ amostras biológicas, ${ }^{44}$ ácidos fracos ${ }^{45}$ e amostras ambientais. ${ }^{46}$

O stacking depende da quantidade de amostra e de base injetadas e do comprimento do capilar. Pode ser usado um sistema com capilares duplos para melhorar este problema.

Necessidade de desenvolvimento de método para analitos diferentes. A escolha dos tampões deve ser criteriosa. Para amostras de baixa condutividade pode ocorrer degradação térmica dos componentes.
Análise de cátions ${ }^{28}$ e ânions, ${ }^{29}$ glutationas, ${ }^{31}$

frações de peptídeos ${ }^{9,11,47}$ e amostras biológicas. . $^{16,30,31}$

Acoplamento em linha da isotacoforese com a CE. ${ }^{48}$ Recomendações para eletrólitos líder e terminador. ${ }^{49,50}$ Transição da ITP para CZE em tITP. ${ }^{49,51}$ Uso de microchips com multicanais, ${ }^{17-19,52-53}$ e detecção por fluorescência a laser. ${ }^{52}$ concentração dos íons. Os picos tornam-se assimétricos, uma vez que a focalização da amostra ocorre apenas em um lado da banda e não em ambos. ${ }^{36}$

Outro problema com amostras de baixa condutividade é a geração de calor excessivo na banda da amostra (devido ao alto campo elétrico) que pode degradar termicamente alguns ou todos os componentes. ${ }^{36}$ Este efeito é minimizado introduzindo-se no capilar volumes de $\mathrm{OH}^{-}$e $\mathrm{H}^{+}$que não formem bandas muito longas.

\section{COMENTÁRIOS GERAIS}

As estratégias de pré-concentração baseadas na velocidade eletoforética do analito são facilmente realizadas on-line e induzem a um ganho de sensibilidade que as torna apreciáveis para diversos tipos de análises. Isto torna possível realizar separações eletroforéticas empregando o detector por absorção no ultravioleta, que é o modo de detecção mais comumente encontrado em equipamentos comerciais. A escolha da estratégia vai depender do tipo de analito e do aumento de sensibilidade necessário para execução da análise. A Tabela 1 apresenta os comentários gerais e as aplicações para as estratégias de aumento de sensibilidade baseadas na manipulação da velocidade eletroforética do analito.

Entre as estratégias mostradas, a que apresenta a maior simplicidade com maior aumento no sinal é o stacking em campo amplificado. Os ganhos são comparáveis ao stacking em grande volume, porém, sem a necessidade de reverter a polaridade, que pode levar à perda de amostra e diminuição da pré-concentração. Já o sucesso da isotacoforese transitente se deve à escolha criteriosa dos elétrolitos líder e terminador, o que não é tão simples, considerando o número de eletrólitos comercialmente disponíveis. Simulações computadorizadas do aumento de concentração obtido através desta estratégia empregando diferentes arranjos de eletrólitos líder e terminador já se encontram disponíveis na literatura, ${ }^{53}$ o que torna o processo de escolha bem mais fácil.

\section{AGRADECIMENTOS}

À Fapesp pelo auxílio concedido.

\section{REFERÊNCIAS}

1. Ewing, G. W.; Analytical Instrumentation Handbook, $2^{\text {nd }}$ ed., Marcel Dekker Inc.: New York, 1997.

2. Tavares, M. F. M.; Quim. Nova 1996, 19, 173.

3. Heiger, D. N.; High Performance Capillary Electrophoresis: An Introduction, $2^{\text {nd }}$ ed., Hewlett-Packard Company: France, 1992.

4. Albin, M.; Grossman, P. D.; Moring, S. E.; Anal. Chem. 1993, 65, 489A.

5. Guzman, N. A.; Capillary Electrophoresis Technology: Chromatographic Science Series, Dekker: New York, 1993. 
6. Chien, R.; Burgi, D. S.; Anal. Chem. 1992, 64, 489A.

7. Chien, R. L.; Burgi, D. S.; J. Chromatogr. 1991, 559, 141.

8. Chien, R. L.; Burgi, D. S.; Anal. Chem. 1992, 64, 1046.

9. Schwer, C.; Lottspeich, F.; J. Chromatogr. 1992, 623, 345.

10. Friedberg, M. A.; Hinsdale, M.; Shihabi, Z. K.; J. Chromatogr., A 1997, 781,35 .

11. Weinberger, R.; Practical capillary electrophoresis, Academic Press, Inc.: San Diego, 1993.

12. Chien, R. L.; Burgi, D. S.; J. Chromatogr. 1991, 559, 153.

13. Osbourn, D. M.; Weiss, D.; Lunte, C. E.; Electrophoresis 2000, 21, 2768.

14. Quirino, J. P.; Kim, J.; Terabe, S.; J. Chromatogr., A 2002, 965, 357.

15. Quirino, J. P.; Terabe, S.; J. Chromatogr., A 2000, 902, 119.

16. Mayboroda, O. A.; Neusuß, C.; Pelzing, M.; Zurek, G.; Derks, R.; Meulenbelt, I.; Kloppenburg, M.; Slagboom, E. P.; Deelder, A. M.; J. Chromatogr, A 2007, 1159, 149.

17. Szökő, É.; Tábi, T.; Haláz, A. S.; Pálfi, M.; Magyar, K.; J. Chromatogr., A 2004, 1051, 177.

18. Takayanagi, T.; Ishida, M.; Mbuna, J.; Driouich, R.; Motomizu, S.; J. Chromatogr., A 2006, 1128, 298.

19. Horáková, J.; Petr, J.; Maier, V.; Znaleziona, J.; Stanová, A.; Marák, J.; Kaniansky, D.; Sevcík, J.; J. Chromatogr., A 2007, 1155, 193.

20. Mikkers, F. E.; Everaerts, F. M.; Verheggen, T. P. M.; J. Chromatogr. 1979, 169, 11.

21. Burgi, D. S.; Chien, R. L; Anal. Chem. 1991, 63, 2042.

22. Shihabi, Z. K.; J. Chromatogr., A 1996, 744, 231.

23. Baker, D. R.; Capillary Electrophoresis, John Wiley: New York, 1995.

24. Chien, R.; Helmer, J. C.; Anal. Chem. 1991, 63, 1354.

25. Shao-Wen, S.; Hua-Ming, T.; J. Pharm. Biomed. Anal. 2004, 36, 43.

26. Macia, A.; Borrull, F.; Calull, M.; Aguilar, C.; Trends Anal. Chem. 2007, 26, 133.

27. Vinther, A.; Soeberg, X. H.; J. Chromatogr. 1991, 27, 559

28. Park, S.; Lunte, C. E.; J. Microcolumn Sep. 1998, 10, 511.

29. Zhao, Y.; Lunte, C. E.; Anal. Chem. 1999, 71, 3985.

30. Ward, E. M.; Smyth, M. R.; O'Kennedy, R.; Lunte, C. E.; J. Pharm. Biomed. Anal. 2003, 32, 813.

31. Hoque, M.; Arnett, S. D.; Lunte, C. E.; J. Chromatogr., B 2005, 827, 51.
32. Simpson Jr., S. L.; Quirino, J. P.; Terabe, S.; J. Chromatogr., A 2008 , 1184,504

33. Church, M. N.; Spear, J. D.; Russo, R. E.; Klunder, G. L.; Grant, P. M.; Andresen, B. D.; Anal. Chem. 1998, 70, 2475.

34. Verheggen, T. P. E. M.; Schoots, A. C.; Everaerts, F. M.; J. Chromatogr. 1990, 503, 345.

35. Tavares, M. F. M.; Quim. Nova 1997, 20, 493.

36. Krivankova, L.; Gebauer, P.; Bocek, P.; J. Chromatogr., A 1995, 716, 35.

37. Macia, A.; Borrull, F.; Calull, M.; Aguilar, C.; J. Chromatogr., A 2006, 1117, 234.

38. Šafra, J.; Pospíśilová, M.; Kavalírová, A.; J. Pharm. Biomed. Anal. 2006, $41,1022$.

39. Tsai, C. H.; Tsai, C. C..; Liu, J. T.; Lin, C. H.; J. Chromatogr., A 2005, 1068, 115.

40. Chou, Y. W.; Huang, W. S.; Chen, C. C.; Lin, S. J.; Wu, H. L.; Chen, S. H.; J. Chromatogr., A 2005,1087,189.

41. Shihabi, Z. K.; J. Chromatogr., A 2005,1066, 205.

42. Mc Grath, G.; Smyth, W. F.; J. Chromatogr., B 1996, 681, 125.

43. Puig, P.; Borrul, F.; Calull, M.; Benavente, F.; Sanz-Nebot, V.; Barbosa, J.; Aguilar, C.; Anal. Chim. Acta 2007, 587, 208.

44. Maeso, N.; Cifuentes, A.; Barbas, C.; J. Chromatogr., B 2004, 809, 147.

45. Chun, M.; Chung, D.; Anal. Chim. Acta 2003, 491, 173.

46. Huang, H-Y.; Chiu, C-W.; Sue, S-L.; Cheng, C-F.; J. Chromatogr., A 2003, 995, 29.

47. Aebersold, R.; Morrison, H. D.; J. Chromatogr. 1990, 516, 79.

48. Kaniansky, D.; Marak, J.; J. Chromatogr. 1990, 498, 191.

49. Krivankova, L.; Gebauer, P.; Thormann, W.; Mosher, R. A.; Bocek, P.; J. Chromatogr. 1993, 638, 119.

50. Krivankova, L.; Bocek, P.; J. Chromatogr., B 1997, 689, 13.

51. Krivankova, L.; Pantucková, P.; Bocek, P.; J. Chromatogr., A 1999, 838, 55.

52. Yongwon, J.; Choi, K.; Kang, M. K.; Chun, K.; Chung, D. S.; Sens. Actuators, B 2005, 104, 269.

53. Schwer, C.; Gas, B.; Lottspeich, F.; Kenndler, E.; Anal. Chem. 1993, 65, 2168. 